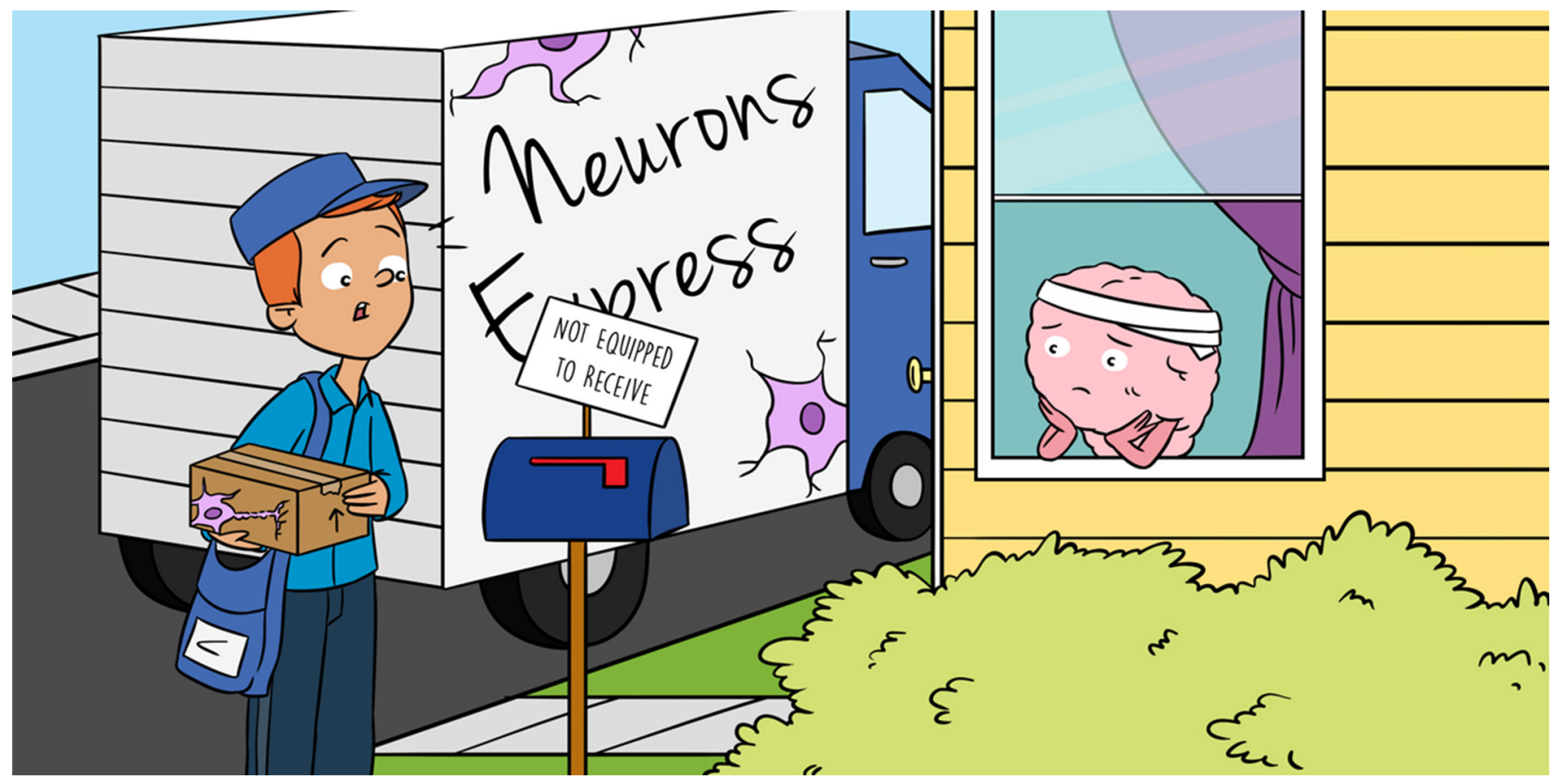

\title{
TUG OF WAR DURING TRAUMATIC BRAIN INJURY
}

\section{Hiba Hasan ${ }^{1,2+}$, Maha Tabet ${ }^{1+}$, Samar Abdelhady ${ }^{1,3}$, Sarah Halabi ${ }^{4}$, Karl John Habashy ${ }^{5}$, Firas Kobeissy ${ }^{1 *}$ and Abdullah Shaito ${ }^{6^{*}}$}

${ }^{1}$ Department of Biochemistry and Molecular Genetics, Faculty of Medicine, American University of Beirut, Beirut, Lebanon

${ }^{2}$ Department of Anatomy and Cell Biology, Justus Liebig University of Giessen, Giessen, Germany

${ }^{3}$ Faculty of Medicine, Alexandria University, Alexandria, Egypt

${ }^{4}$ Biology Department, Faculty of Arts and Sciences, American University of Beirut, Beirut, Lebanon

${ }^{5}$ Faculty of Medicine, American University of Beirut, Beirut, Lebanon

${ }^{6}$ Department of Biological and Chemical Sciences, Lebanese International University, Beirut, Lebanon

\section{YOUNG REVIEWER:}

Human behavior depends on the cooperation of about 100 billion brain cells, called neurons. The generation of new neurons occurs through a process called neurogenesis. Previously, scientists thought that neurogenesis stopped before birth. However, scientists have recently found that neurogenesis can still occur after birth and continues throughout life. Injuries to the brain can lead to the death of neurons, which is called neurodegeneration. Hence, one-time severe damage or repeated smaller injuries to brain neurons can lead to serious diseases called neurodegenerative disorders. Neurogenesis is important to replace damaged neurons, especially after brain injury. Scientists try to find ways to decrease the adverse effects of brain injury. One way is to help the brain to make more neurons following injury, by enhancing neurogenesis. This can help to treat brain injuries and neurodegenerative diseases. 
Figure 1

Sites of neurogenesis in the brain. This figure shows an adult human brain where neurogenesis, or the formation of new neurons, occurs in two regions. These two regions are the hippocampus and the sub-ventricular zone (shown in blue). In the hippocampus, neurogenesis specifically occurs in an area called the dentate gyrus. One way to detect newly formed neurons is to use BrdU staining. BrdU becomes part of the DNA of a new cell and can then be seen under the microscope using specific detector molecules. The picture in the upper left shows the neurons from the hippocampus of a mouse. The mature, old neurons show a green color, while the new neurons show an additional red color because they contain BrdU in their DNA.

\section{NEURONS}

Are fundamental cells of the brain that transmit information to other cells.

\section{NEURAL STEM CELL}

Are cells that can renew themselves and can form new neurons.

\section{NEUROGENESIS}

A process by which new neurons are produced

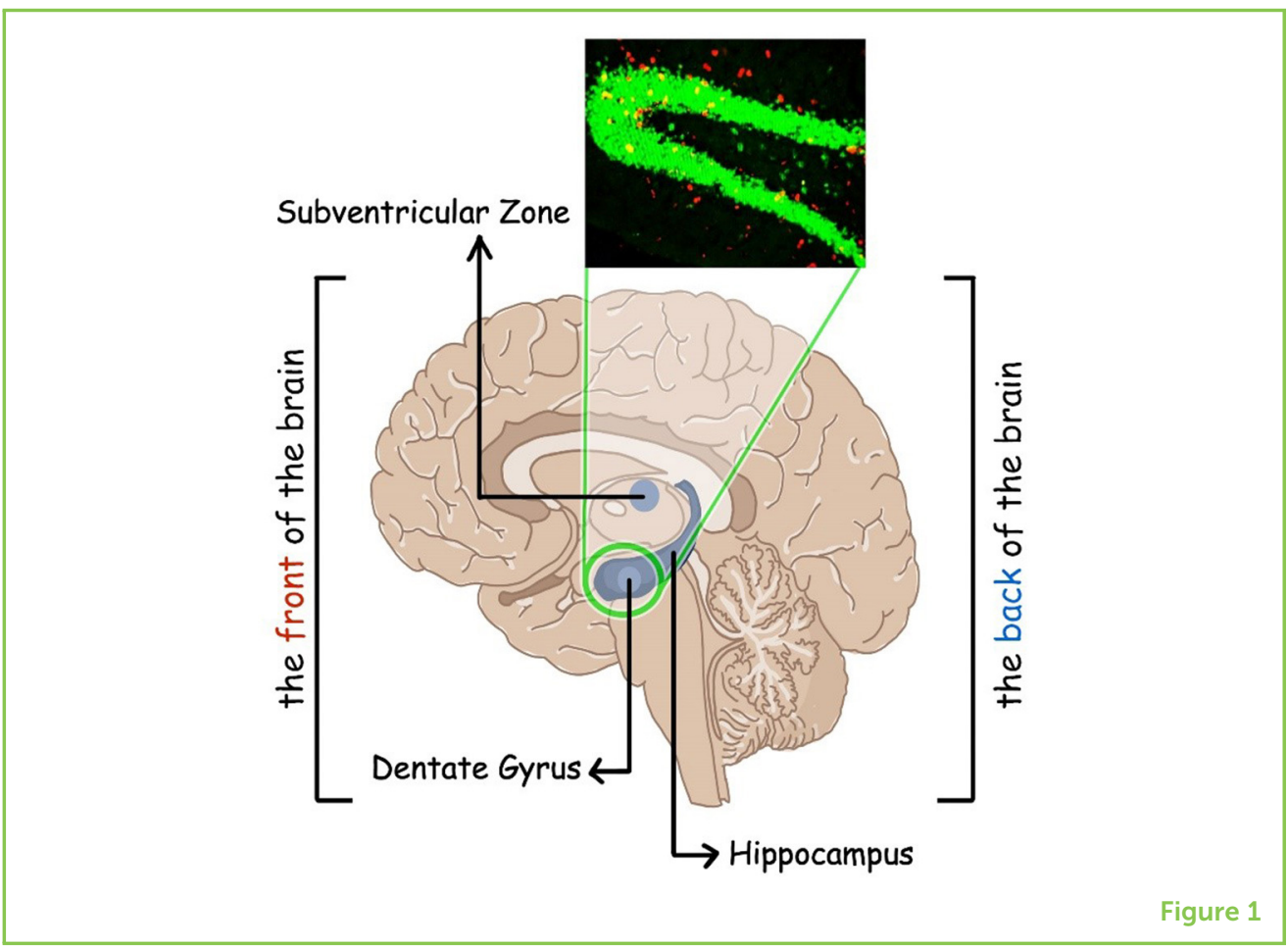

\section{NEURAL STEM CELLS: THE BENCH PLAYERS}

The brain is the most complex organ in the body. Our brains allow us to think, observe, analyze, move, feel, and do many other tasks. Like other organs, the brain is made up of several types of cells. Neurons are the main cells of the brain. They are considered the main players in producing the wonderful range of human behavior. A neuron connects with other neurons to transmit messages. This message transmission allows us to do all the things we do. The brain also consists of other types of cells with different jobs, such as supporting and nourishing the neurons, helping the neurons to transmit their signals, or defending the brain against foreign organisms [1].

Not long ago, scientists believed that no new neurons could join the "team" of brain cells once it was formed before birth-it was thought that new neurons were not made after a person was born. Later, scientists discovered that two areas in the brain could make new neurons. These two brain areas contain special cells called neural stem cells (NSCs), which can generate new neurons through a process called neurogenesis. The two areas of the brain that contain NSCs and can perform neurogenesis throughout life are: (1) the sub-ventricular zone, which is the area of the brain where most of the neurogenesis happens; and (2) a region in the hippocampus, which is the part of the brain responsible for memory (Figure 1). Interestingly, it has also been found that NSCs can act as "bench players" that join the brain-cell team in case of injury, meaning that neurogenesis increases following damage to the brain [2]. 


\section{HOW DO WE SPOT THE NEWBIES?}

There are several ways to spot the neurons that have recently joined the team. BrdU staining is one method used to detect the new neurons, which are usually produced by NSCs. BrdU is a chemical that can be added to brain cells in the lab and then it becomes incorporated into the DNA of new neurons. BrdU becomes a part of and marks the DNA of new cells only, and the mature older cells do not get marked by BrdU. The staining by BrdU molecules in new cells can be detected under a microscope (Figure 1).

Another strategy to find newly formed neurons is to look for their mascot. Let us pretend that each type of cell in the brain has a specific mascot. If we can spot the mascot, then we will know the team and the team is the type of brain cell! But, what is the mascot of a neuron? Newly formed neurons will have specific molecules that are made only by them (their mascot). What scientists do is to look for the presence of these specific molecules. To find these specific molecules, scientists then use specific detector molecules that stick only to the mascot of new neurons and not to other mascots. The detector molecules can be seen under a special microscope.

Yet another method is to determine the age of the new neurons. This is possible because new neurons are much younger than the neurons that you were born with. Scientists do this by looking at the carbon content of neurons. Carbon is an element found in nature and is a building block of everything in life, including cells. The properties of carbon change over time, and we can know how old something is by looking at the type of carbon it has. Think of it this way: every year, the newcomers get a new bracelet as a welcome gift, which differs from bracelets distributed in the previous years. So, we can tell which year a member joined the team by looking at their bracelets. The bracelets are the type of carbon they have [2].

\section{BRAIN INJURY AND NEURODEGENERATION}

Most of us have bumped our heads once or twice in our lifetimes. We may have felt some sort of pain, but woken up the next day as if nothing happened. This is known as a head injury. Head injuries usually do not lead to long-term consequences. But, if the hit to the brain was repeated or if the initial bump was very severe, head injury can lead to

TBI

An injury to the brain that disrupts normal brain functioning. a traumatic brain injury ( $\mathrm{TBI})$. The worse the $\mathrm{TBI}$, the more serious the outcome will be for the injured person and the more changes will occur in that person's brain.

There are two stages to TBI, called primary injury and secondary injury (Figure 2). The primary injury involves changes in the brain that occur immediately after hitting our heads. There will be damage to cells, 
Figure 2

Events that occur following TBI.

Traumatic brain injury consists of two phases: the primary injury and the secondary injury. The primary injury takes place within seconds to minutes following the onset of TBI. Primary injury involves direct damage to neurons caused by the blow to the head. The secondary injury takes place later, within minutes to weeks following the injury. The secondary injury involves the release of inflammatory molecules by microglia and other immune cells. In severe cases, microglia and other immune cells release a lot of inflammatory molecules during the secondary injury, which can lead to neuron death.

\section{INFLAMMATION}

It is a protective biological response that starts under harmful conditions, such as stress. It is one way your body fights infection, injury, or disease. It involves immune cells, blood vessels, and many molecules inside the cell.

\section{MICROGLIA}

A type of immune cell that is found in the brain.

\section{NEURODEGENERATION}

A process that causes neurons to die. It is a measure of neuron death.

\section{NEURODEGENERATIVE} DISEASES

Neurodegenerative diseases are a set of

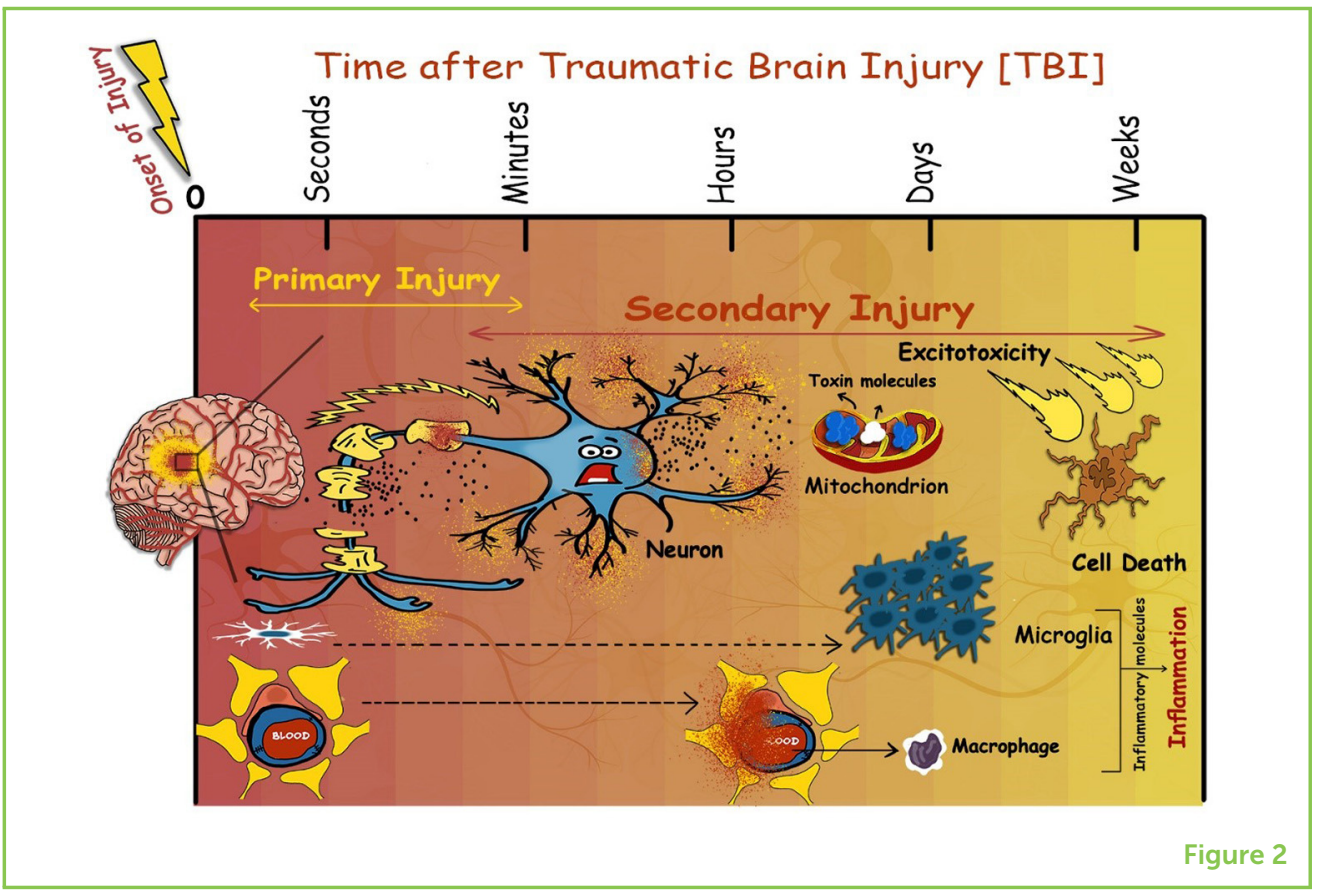

bleeding near the area of injury, and pain. Hours or even days after the primary injury, the secondary injury takes place. The secondary injury involves more changes in the brain, including excitotoxicity (when neurons are damaged or even killed because of being highly active) and inflammation. Inflammation of neurons involves the activation of the immune cells of the brain that are called microglia. Activated microglia release inflammatory molecules, which recruit other types of immune cells to the location of the brain injury, thus increasing the inflammation even more. This increase in inflammation is a normal response to injury and is vital to the maintenance of health. However, an uncontrolled increase of inflammation is harmful to cells.

In addition, during the secondary injury, brain cells become stressed and start to accumulate toxin molecules that can eventually lead to the death of neurons, or what we call neurodegeneration. Therefore, it is often the secondary injury that causes the most damage and neurodegeneration, even though the actual hit to the head took place days or weeks earlier. The death of many neurons can be very dangerous and even lead to long-term problems called neurodegenerative diseases [3]. Scientists are trying to decrease the bad outcomes of TBI by increasing neurogenesis after the injury.

\section{NEUROGENESIS AND NEURODEGENERATION: TUG OF WAR}

Injury to the brain, as mentioned above, causes inflammation. Inflammation in the brain is caused by the activation of microglia and other immune cells called macrophages. These cells secrete 
Figure 3

Balance between neurogenesis and neurodegeneration following TBI. The balance between neurogenesis and neurodegeneration is determined by the environment caused by the injury. In mild TBI, there is a balance between inflammatory molecules that favor neurogenesis and other inflammatory molecules that favor neurodegeneration. The inflammatory molecules that favor neurodegeneration act as toxin molecules that kill neurons. As a result in the cases of severe or repeated $\mathrm{TBI}$, the balance is disrupted and tips more toward neurodegeneration, since the production of toxin molecules is highly increased.

diseases characterized by the progressive loss of neurons or neural function. They lead to problems with movement, or mental functioning. Most of these diseases are not curable.

\section{MACROPHAGE}

A type of immune cell involved in the process of inflammation.

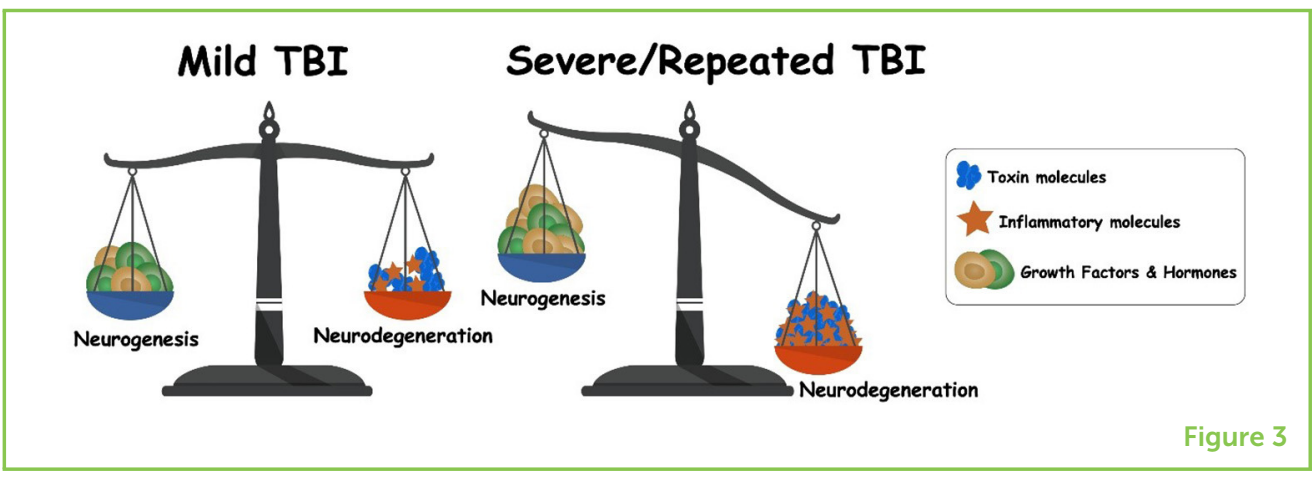

chemicals, such as the inflammatory molecules, that can promote either neurogenesis or neurodegeneration (Figure 3). But, how?

If the brain injury is very mild, controlled inflammation in the brain will occur. This controlled inflammation has a positive effect on neurogenesis, since it aims to replace the lost neurons. However, if the injury to the brain is very severe or if the brain injury is repeated, as often seen in certain sports activities, then this can lead to severe inflammation. Severe inflammation cannot be controlled. Some inflammatory molecules released during severe inflammation have a negative effect on neurogenesis and form a harsh environment for growth of new neurons. In the presence of these inflammatory molecules, even if newborn neurons form then they cannot survive. So, in the case of severe or repeated head injuries, there will be more neurodegeneration than neurogenesis.

This situation is like weighing two things on a pan balance. If there are equal weights on both sides, the balance is at equilibrium. This is what happens in the case of controlled inflammation: the amount of neurogenesis that occurs is somewhat equal to the amount of neurodegeneration that took place as a result of the injury. However, if one side of the balance is heavier than the other, this will cause the balance to tilt. In the case of severe brain injury, there is uncontrolled inflammation. This causes the amount of neurodegeneration to be greater than the amount of neurogenesis (Figure 3). In this situation, the balance tilts toward neurodegeneration and the secondary injury can lead to serious neurodegenerative diseases [4].

\section{THE EFFECTS OF NEURODEGENERATION}

Neurons work together to perform all brain functions. If neurons start to die, the functions of the brain are affected. People who suffer a mild or a moderate TBI may lose a few neurons. They may experience problems with their thinking or memory, or with their ability to pay attention. 
Severe TBI occurs when the brain receives a severe injury, for example, during car crashes or hard falls. People who perform contact sports, such as football players, hockey players, soccer players, and boxers are examples of people who may be exposed to severe TBI or repeated TBI.

During severe $\mathrm{TBI}$, many neurons die and this causes neurodegeneration to outweigh neurogenesis. Severe TBI or repeated hits on the head may put people at risk of developing neurodegenerative diseases because a great number of neurons die.

Neurodegenerative diseases can show up tens of years following TBI. Neurodegenerative diseases include Alzheimer's disease, which causes memory loss, and Parkinson's disease, in which people start to shake because they lose control of their muscles. Amyotrophic lateral sclerosis (ALS), a disease in which people lose control of their muscles, and chronic traumatic encephalopathy are other types of neurodegenerative diseases. Encephalopathy refers to diseases that affect the function or structure of the brain and in chronic traumatic encephalopathy the patients show problems in their behavior, mood, and thinking, leading to confusion and forgetfulness.

\section{CONCLUSION}

TBls should always be taken seriously. The damage that occurs following TBI is not always visible immediately. If you experience a TBI, it can affect you in the long run. There are many factors that influence the outcome of TBI. If the injury is very severe, neurogenesis is not as effective, and this can shift the balance toward neurodegeneration. So, you should always protect your head when doing dangerous activities or when playing sports. Protecting your brain is a must $[3,5]$ !

\section{REFERENCES}

1. Purves, D., Augustine, G. J., Fitzpatrick, D., Katz, L. C., LaMantia, A. S., McNamara, J. O., et al. (Eds.). 2001. "Neuroglial cells," in Neuroscience, 2nd Edn.

(Sunderland, MA: Sinauer Associate). p. 1-43.

2. Ming, G. L., and Song, H. 2011. Adult neurogenesis in the mammalian brain: significant answers and significant questions. Neuron 70:687-702. doi: 10.1016/j.neuron.2011.05.001

3. Kobeissy, F., Mondello, S., Tumer, N., Toklu, H. Z., Whidden, M. A., Kirichenko, N., et al. 2013. Assessing neuro-systemic \& behavioral components in the pathophysiology of blast-related brain injury. Front. Neurol. 4:186. doi: 10.3389/fneur.2013.00186

4. Shohayeb, B., Diab, M., Ahmed, M., and Ng, D. C. H. 2018. Factors that influence adult neurogenesis as potential therapy. Transl. Neurodegener. 7:4. doi: 10.1186/s40035-018-0109-9 
5. Abou-Abbass, H., Bahmad, H., Ghandour, H., Fares, J., Wazzi-Mkahal, R., Yacoub, B., et al. 2016. Epidemiology and clinical characteristics of traumatic brain injury in Lebanon: a systematic review. Medicine (Baltimore) 95:e5342. doi: 10.1097/MD.0000000000005342

SUBMITTED: 31 October 2019; ACCEPTED: 04 August 2020; PUBLISHED ONLINE: 30 September 2020.

EDITED BY: Robert T. Knight, University of California, Berkeley, United States

CITATION: Hasan H, Tabet M, Abdelhady S, Halabi S, Habashy KJ, Kobeissy F and Shaito A (2020) Tug of War During Traumatic Brain Injury. Front. Young Minds 8:119. doi: 10.3389/frym.2020.00119

CONFLICT OF INTEREST: The authors declare that the research was conducted in the absence of any commercial or financial relationships that could be construed as a potential conflict of interest.

COPYRIGHT @ 2020 Hasan, Tabet, Abdelhady, Halabi, Habashy, Kobeissy and Shaito. This is an open-access article distributed under the terms of the Creative Commons Attribution License (CC BY). The use, distribution or reproduction in other forums is permitted, provided the original author(s) and the copyright owner(s) are credited and that the original publication in this journal is cited, in accordance with accepted academic practice. No use, distribution or reproduction is permitted which does not comply with these terms.

\section{YOUNG REVIEWER}

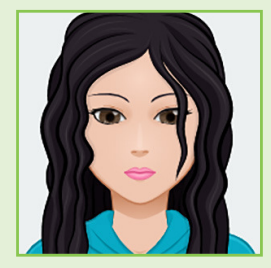

\section{EUNBI, AGE: 13}

My name is Eunbi and I am from San Francisco. I am currently in eighth grade. Reading science articles and discovering further wonders about the world and mechanisms of human diseases are very fascinating for me. I believe that even small discoveries could influence the future of this world.

\section{AUTHORS}

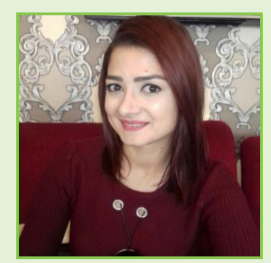

\section{HIBA HASAN}

I am currently a volunteer in the Biochemistry and Molecular Genetics department at the American University of Beirut. The lab focuses on understanding the pathological basis of brain injuries and tests cell- and drug-based therapies for traumatic brain injuries. I have a Master's in Biology with a specialization in Immunology, where I tested the anti-inflammatory and antioxidant activities of different natural agents on autoimmune diseases. I hope to pursue a Ph.D. focusing on the immunopathological basis of autoimmune disorders and hope to find a cure for such diseases. 

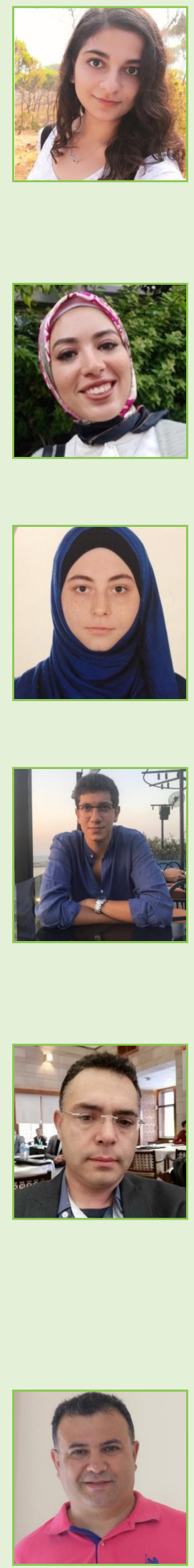

\section{MAHA TABET}

I acquired an M.Sc. in Neuroscience from the Neuroscience Research Centre at the Lebanese University and conducted my Master's research project in the department of Biochemistry and Molecular Genetics at the American University of Beirut (AUB). My project examined the role of mitochondria and oxidative stress in traumatic brain injury (TBI), with a focus on testing a possible antioxidant treatment. In addition to my love for research, I like reading, drawing, and writing.

\section{SAMAR ABDELHADY}

I am a medical doctor. I did a research internship in Dr. Kobeissy's lab at the American University of Beirut (AUB) to study traumatic brain injury [TBI] using animal models. In my free time, I love drawing scientific illustrations and brain art, watching movies that talk about the brain, and since I work for long hours at a desk, I enjoy going hiking to keep my body fit.

\section{SARAH HALABI}

I graduated from Lebanese International University with a degree in biochemistry. I am currently studying for my Master's degree in Biology at the American University of Beirut. My research interests include cancer, transcriptomics, proteomics, and microbiology. I want to eventually complete a Ph.D. in Biology or Biochemistry.

\section{KARL JOHN HABASHY}

I am a medical student at the American University of Beirut, where I have earned my Bachelor of Sciences with distinction. During my undergraduate years, I was trained in Dr. Noel Ghanem's lab, which focuses on mechanisms of neurogenesis and its implications on neurodegenerative diseases. During my ongoing medical studies, I volunteer in Dr. Firas Kobeissy's lab and study traumatic brain injuries. I plan to pursue a career both as a physician and a researcher in the field of neurosciences.

\section{FIRAS KOBEISSY}

I am a neuroscientist with extensive experience in experimental brain injury. I am an assistant Professor at the Department Biochemistry at the American University of Beirut. I obtained my Ph.D. from the University of Florida in the area of neuroscience. My current research focuses on identifying biomarkers for drug abuse toxicity and traumatic brain injury neuroproteomics. I am a member of the Center of Neuroproteomics and Biomarker Research and the Center for Traumatic Brain Injury Studies at the McKnight Brain Institute at the University of Florida. *firasko@gmail.com

\section{ABDULLAH SHAITO}

I obtained my Ph.D. at the University of Texas Southwestern at Dallas (Texas, USA), where I studied microbiota interaction with intestinal cells. Currently, I am an Assistant Professor at the Lebanese International University (Beirut, Lebanon). The current research projects I am working on include the use of stem cells to treat $\mathrm{TBI}$, and the cell-cell communication that happens in different cancers. I also teach several core Biology courses to undergraduates. Email me when me when you get into college and need help in your biology courses. *abdshaito@gmail.com

${ }^{\dagger}$ These authors have contributed equally to this work 